their hosts, but also among various parasitic species, even though they may be related to each other morphologically and taxonomically. Therefore, in biochemical studies of parasites, similarities and differences must receive equal attention and any one-sided view will result in a distorted picture. It is suggested that comparative biochemistry should be concerned with the nature of biochemical variability from patterns common to many forms of life; this, in turn, should contribute to a better understanding of biochemical evolution to a parasitic form of life.

\title{
VIBRATIONS: THEIR EFFECTS AND ANALYSIS
}

$I^{\mathrm{s}}$ this technological age, in which larger and larger amounts of power are being harnessed to aid mankind, it is not surprising that the unwanted adjunct of power, vibration, is becoming of great importance. Thus, for example, it is not only the public itself which is annoyed and fatigued by the noise of jet aircraft, but also the fabric of aeroplanes is now tending to show signs of fatigue and to crack. Again, in the field of supersonic transport, the motion of the aircraft can be of a frequency (around $5 \mathrm{c.} / \mathrm{s}$.) at which the body is least attuned to absorb the vibrational energy imposed upon it.

These topics, together with the more industrial problems of machinery vibration and occupational diseases arising from excessive vibration of drills, etc., were the subjects discussed at the recent meeting of the Acoustics Group of the Physical Society, held on January 20, at the Imperial College of Science and Technology, London.

Dealing first of all with problems of the vibration isolation of machinery, Mr. P. H. Allaway (Absorbit, Ltd.), in a review paper, gave an excellent appraisal of the many types of isolating materials and systems now available. The effect of the rigidity of the ground or floor structure was discussed, together with the effect of damping in shock vibration isolation. He emphasized the need for choosing the right type of installation for any type of application, and showed in the ensuing discussion a wealth of valuable experience and 'know-how'.

Dr. John Agate (Ipswich and East Suffolk Hospital) presented a somewhat frightening picture of the influence of vibrations in industry on the hands of operating personnel. Four diseases were mentioned in all : cysts of carpal bones, osteo-arthritis, softtissue injuries and a circulatory disturbance known as Raynaud's phenomenon. The small cysts can only be seen on X-ray examination and are not of any significance. Osteo-arthritis of the elbows is, of course, not confined to people working with vibrating machinery, though there is ample evidence of this being a contributory cause. Raynaud's phenomenon, which Dr. Agate dealt with extensively, is a circulatory disorder of the blood stream in the hands, and it arises from their operating in an environment of heavy vibration. In such cases, there is a lessening of the blood stream in the small arteries of the hands, and it is typified by the fingers and even the whole hand becoming white or blue. It is difficult to schedule the disease as a disability, since most fingers show these symptoms in the cold; further, many workers using vibrational types of machinery do not suffer with this loss of circulation in the fingers in the warmth of their factories, but only show the symptoms later in colder conditions, and then about six to eighteen months after first exposure.

The prevention of this disease is not easy, since any frequency less than $600 \mathrm{c} . / \mathrm{s}$. causes the damage to the arteries, the range of $40-125 \mathrm{c} . / \mathrm{s}$. nevertheless being the most serious. The lesson to be learnt is, in fact, the need for automation for any processes of this kind. Since this can only be introduced in very special instances, the solution is still unavailable and needs further scientific study.

In view of the recent interest in supersonic transport aircraft, Flight Lieut. Guignard's description of the work of the R.A.F. Institute of Aviation Medicine on physiological effects of low-frequency vibration was both interesting and significant from the point of view of design. The jet liners of the present have a natural frequency of oscillation of the fuselage structure somewhere in the region of 4-6 c./s., and pilots placed in the cockpits of these aircraft have been known to suffer from the up-and-down movements of that part of the long fuselage. The experiments described illustrate the reasons for this, and emphasize the need for designing away from such frequencies. It is found (Fig. 1) that if a male person, in a relaxed sitting posture, is vibrated vertically through the seat, a resonance occurs at some $5 \mathrm{c.} / \mathrm{s}$, the amplification of the head and shoulders being as much as three times that of the seat movement. Above this resonance frequency, the damping in the body eliminates harmonics of higher frequency. The effect of tensing the body is favourable, increasing the resonant frequency to $6 \cdot 3 \mathrm{c} . \mathrm{s}$. and also increasing the damping, thus reducing the amplitude of head movement by a small though significant amount. At these frequencies around $5 \mathrm{c} . / \mathrm{s}$., tests in the United States indicate a serious reduction in performance with poor eye-correlation, fatigue and pains in the chest.

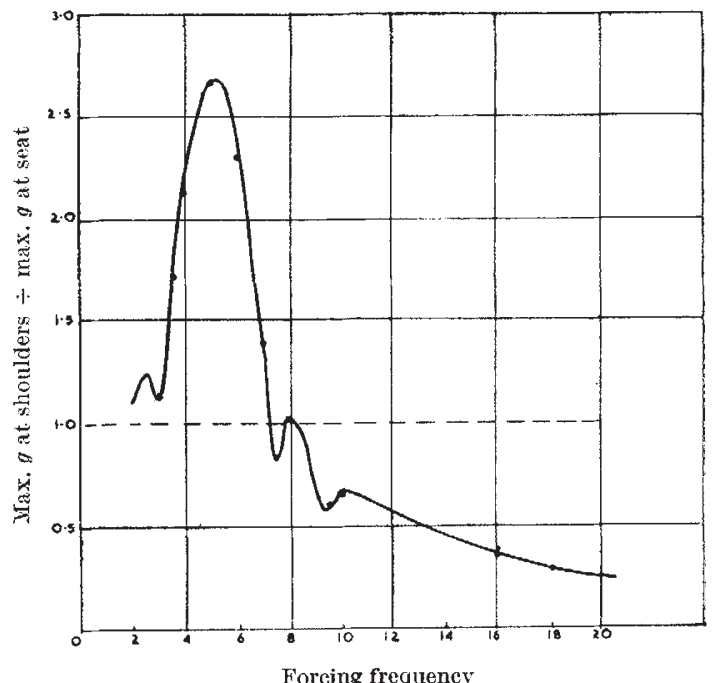

Fig. 1. Ratio of shoulder to seat acceleration plotted against frequency. Note the sharp resonance of the body at $5 \mathrm{c} . / \mathrm{s}$. 
It is clear that this is a phenomenon of vital significance in the design of aircraft. The problem is not confined to aircraft, however. Ships cannot in any way claim freedom from vibrations of this frequency, while even trains, 'juddering' to a standstill in railway stations, set up exactly this type of disturbance.

Two complementary papers concerning the fatigue of aireraft structures due to vibrations set up by jet noise were presented respectively by Prof. E. J. Fichards and Dr. B. L. Clarkson, of the Department of Aeronautics, Univorsity of Southampton. Jet noise arises from the turbulent motion of the jet gas as it mixes with the surrounding air. The total radiated energy is only a very small fraction of the energy of the jet, however (say, a hundredth of 1 per cent), and elaborate methods of experimentation have to be devised to measure the turbulence funct,ions which are directly related to the radiated noise. In his paper, Prof. Richards described the space-time correlator and other apparatus used to obtain the spectrum of turbulence as seen by an observer moving with the jet stream. The relevant speeds of convection were discussed and a first attempt made to predict the radiated sound from the spectra of turbulence measured in this way.

There is, in general, no one discrete frequency of sound emitted from a jet. Consequently, the adjoining structure is vibrated randomly by the noise field and can in theory oscillate in an infinity of modes of oscillation. If a large number of modes do occur in practice, it is clear that the theoretical approach to prediction must be different from that adopted if the vibration is of a relatively simple kind. Dr. Clarkson's paper described experiments carried out on the vibrations of the rear part of the Caravelle airliner due to jet efflux, a programme carried out jointly by the firm, Sud-Aviation, and the University of Southampton. The analysis was concentrated on the strains in the centre of four panels of the fuselage skin aft of the engine nozzle; the first three being in line between two circumferential frames and the fourth being adjacent to the centre gauge of the first group but separated by a frame. Correlation measurements indicated that the lower frequencies (up to $500 \mathrm{c} . / \mathrm{s}$.) were associated with essentially overall vibration modes and have low strain amplitude. At the higher frequencies, having the larger strains, the frames tended to act as boundaries and the mode of vibration was confined to the stringers and panels between any two adjacent frames. In these measurements on this particular aircraft, the main resonance peak in each panel occurs at about $600-700 \mathrm{c} . / \mathrm{s}$. and has been identified with the fundamental stringer twisting mode (that is, adjacent panels are $180^{\circ}$ out of phase). Strain response in the higher frequency range of $800-1,000 \mathrm{c} / \mathrm{s}$. appears to be associated with a considerable number of closely spaced resonant frequencies.

This method of analysis using correlation techniques is a considerable step forward in the study of random vibrations of aircraft, ships and submarines, and the success outlined in the paper suggests that greater use should be made of this method of vibration analysis. $\quad$ E. J. RrCHaRds

\title{
A CONVECTIVE-TURBULENCE THEORY OF THUNDERSTORMS
}

\author{
By Dr. N. RAMALINGAM \\ Director, Regional Meteorological Centre, New Delhi
}

\begin{abstract}
T is well known among meteorologists that in practical forecasting of thunderstorms the methods in current usage, such as the parcel methods, etc., of analysis of the dry-bulb and wet-bulb soundings of the atmosphere plotted on $T-\varphi$ grams or other thermodynamic diagrams, are often unreliable.

The initiation of thunderstorms, at least in such cases that occur in a calm atmosphere over land on hot afternoons, as experienced over India in the hot pre-monsoon months, has been investigated from the point of view of modern knowledge about turbulence. In doing that, however, the limiting condition :
\end{abstract}

$$
\frac{\rho_{1}-\rho_{0}}{\rho_{0}}>\frac{27 \pi^{4} k v}{4 g h^{3}}
$$

given by Lord Rayleigh ${ }^{1}$ from his mathematical investigation of the motion in the Bénard cells formed, when a fluid of thickness $h$ in equilibrium with its density $\rho_{1}$ at the top being greater than its density $\rho_{0}$ at the bottom breaks down into convection, is taken as the main physical basis. In the above expression $v$ is the kinematic coefficient of viscosity, and $k$ is the coefficient of conduction of heat of the fluid.

Indeed, even so far back as 1925, Brunt ${ }^{2}$ made it very clear that some of the phenomena associated with thunderstorms can be explained in the same way, and that the violence of thunderstorms would appear to indicate that a high degree of instability is built up before the breakdown occurs. This suggestion, however, does not appear to have been pursued quantitatively.

In his original paper, Rayleigh gave the limiting condition as :

$$
\beta^{\prime} r>\frac{27 \pi^{4} k \nu}{4 h^{4}}
$$

where $\beta^{\prime}(=-\beta)$ is the lapse-rate; and $r=g \alpha, x$ is the coefficient of expansion of the fluid, and $g$ is the acceleration due to gravity.

Since he was considering a fluid of small thickness $h$, he took :

$$
\beta^{\prime}=-\beta=\frac{\Theta_{1}-\Theta_{0}}{h}
$$

$\Theta$ being the departure from the equilibrium tem. perature, and :

$$
r=g \alpha_{0}=g\left(\rho_{1}-\rho_{0}\right) / \rho_{0}\left(\Theta_{1}-\Theta_{0}\right)
$$

hence, (2) reduces to (1).

If, however, we take a layer of air of thickness $z$, then we may take $\beta^{\prime}=\frac{d \Theta}{d z}$; and from the relation $p_{1}=p(1+\alpha \mathrm{d} \Theta)$ and taking $p_{1}-p=\mathrm{d} \rho$, we may 